a short survey capturing demographics, social media use and interest in future testing.

Results The advertisement received 401732 impressions" (displays of the advertisement to users). Nearly all $98 \%$ ) impressions were to 19-24-year-olds. The daily impressions varied enormously, from 0 to more than 100000 . In the first week, although the advertisement received up to 2,900 daily impressions, no one clicked it. We gradually increased our "bid per click" - the amount we agreed to pay Facebook for each click一from $\$ 0.62$ to $\$ 0.90$. We also modified the advertisement format to depict a college student and to clearly specify the $\$ 25$ cost of the test. Following these changes, both the number of impressions and "clicks" rose dramatically. Seventy-five individuals clicked the advertisement ( 25 women and 50 men), with an average cost per click of $\$ 0.84$. Three students, all female, came for chlamydia testing; one tested positive. Among surveyed individuals $(n=60), 75 \%$ reported using Facebook daily or more often. Few $(10 \%)$ reported ever clicking on Facebook advertisements. Only one surveyed student noticed our Facebook advertisement. The direct-to-laboratory testing concept was popular, with $75 \%$ reporting willingness to use this service in the future.

Conclusions In this short pilot study, 75 individuals clicked on a Facebook advertisement about chlamydia testing, three came for testing and one tested positive. Facebook advertisements are inexpensive, with high exposure to the target population. A small increase in bid per click can make a very large difference in total impressions. The format of the advertisement is critical for catching the attention of the target audience, because many students report never noticing or clicking Facebook advertisements.

\section{5-S1.05 COMPUTER ASSISTED SELF INTERVIEWING IN A SEXUAL HEALTH CLINIC AS PART OF ROUTINE CLINICAL CARE: IMPACT ON SERVICE AND PATIENT AND CLINICIAN VIEWS}

doi:10.1136/sextrans-2011-050109.155

\author{
${ }^{1} \mathrm{~L}$ Vodstrcil, ${ }^{1} \mathrm{~J}$ Hocking, ${ }^{2} \mathrm{R}$ Cummings, ${ }^{1} \mathrm{M}$ Chen, ${ }^{1} \mathrm{C}$ Bradshaw, ${ }^{1} \mathrm{~T}$ Read, ${ }^{2} \mathrm{~J}$ Sze, \\ ${ }^{1} \mathrm{C}$ Fairley. ${ }^{1}$ The University of Melbourne, Parkville, Australia; ${ }^{2}$ Melbourne Sexual Health \\ Centre, Carlton, Australia
}

Background Computer assisted self interviewing (CASI) has been used at the Melbourne Sexual Health Centre (MSHC) in Victoria, Australia since June 2008, to obtain a pre-consultation sexual risk history. We aimed to evaluate the impact of CASI on consultation times, STI testing rates, patient response rates to CASI questions, and obtain patient and clinician views on CASI.

Methods The proportion of patients who declined to answer questions using CASI since 2008 was calculated. We then used the same 12 week period (Feb to May) over three years (2008 pre-CASI), (2009 CASI period), and (2010 non-CASI period-due to a computer theft) to assess consultation times and STI-testing rates. We carried out surveys of clinicians and patients to determine their experience and the acceptability of CASI as part of routine clinical practice.

Results 14190 patients completed CASI during the audit period. Men were more likely than women to decline questions about the number of partners they had of the opposite sex $(4.4 \% \mathrm{v} 3.6 \%$, $p=0.05)$ and same sex $(8.9 \% \vee 0 \%, p<0.01)$. One third $(34 \%)$ of HIVpositive men did not answer questions on number of partners and $18 \%$ declined questions about condom use with insertive anal sex. There was no difference in the mean consultation times during CASI and non-CASI operating periods ( $p$ ?0.17). Only the proportion of women tested for chlamydia differed between CASI and nonCASI periods $(p<0.01,84 \% \vee 88 \%$ respectively). 267 patients completed the survey about CASI. Most $(72 \%$ men and $69 \%$ women) were comfortable using the computer and reported that all their answers were accurate $(76 \%$ men and $71 \%$ women). Half preferred CASI but $18 \%$ would have preferred a clinician to have asked the questions. 39 clinicians completed the staff survey, but a varying proportion $(11 \%-44 \%)$ felt that face-to-face interviewing was more accurate, depending on the risk factor. Only 5\% were unsatisfied with CASI.

Conclusions This is the first evaluation of CASI operating routinely in a sexual health clinic. We have demonstrated that CASI is acceptable to most patients and clinicians in a sexual health setting and does not adversely affect various measures of clinical output. The true value of CASI is most likely to be realised when it is integrated with further innovations in clinical care such as the development of express clinical services, decision support software and detailed behavioural surveillance system.

\section{5-S1.06 STD CLINIC TRIAGE BASED ON COMPUTER-ASSISTED SELF INTERVIEW: THE KING COUNTY EXPERIENCE}

doi:10.1136/sextrans-2011-050109.156

J Dombrowski, M Golden. University of Washington \& Public Health, Seattle \& King County HIVISTD Program, Seattle, USA

Background Triaging patients to different service levels based on responses to computer-assisted self interview (CASI) could increase the cost-efficiency of providing care in STD clinics.

Methods In October 2010 we began having all patients at the King County STD Clinic provide their medical and sexual histories using CASI. Patients are triaged to express or routine care with a computer algorithm based on CASI data. The express care protocol is to collect specimens for HIV/STD testing without physical examination or counselling. Prior to implementation, we conducted 2 months of parallel data collection in which patients who completed CASI were interviewed by clinicians blinded to CASI results. We used $\kappa$ statistics to compare CASI and clinician-obtained data. We tracked visit times for walk-in visits in two 10-day periods pre- and postimplementation, and attempted to survey all patients who used CASI on 10 days about interview and visit type preferences.

Results 875 patients completed both CASI and clinician interviews on 33 days (see Abstract O5-S1.06 table 1). Four months postimplementation, 2731 patients had completed CASI, 420 (15\%) of whom were triaged to express care. Common reasons for triage to routine care were (mutually exclusive): symptoms ( $72 \%$ of routine care), contact to HIV/STD or a symptomatic partner (12\%), and needing a vaccine $(12 \%)$. Patients triaged to express care had lower rates of gonorrhoea $(0.7 \%$ vs $5.7 \%, p<0.001)$, chlamydial infection (3.0\% vs $7.5 \%, p<0.001)$ and syphilis $(0 \%$ vs $1.3 \%, p=0.02)$ than those triaged to routine care. Chart review and informal qualitative data indicated that clinician adherence to the express care protocol varied. Mean visit times were 32 (SD 3) min pre-implementation and 31 (SD 3) min post-implementation. 133 (39\%) of 337 patients completed surveys. $106(80 \%)$ either preferred CASI to clinician interview or had no preference; 27 (59\%) of 46 asymptomatic patients preferred express care.

Conclusions Our CASI system collected accurate data on key aspects of the history, effectively triaged patients at lower risk for bacterial STD diagnosis and was acceptable to most patients surveyed. Lack of acceptability among some clinicians and poor patient recall of vaccine history were barriers to full implementation of the triage system. To date, CASI triage has not improved clinic efficiency as measured by visit time. Our experience suggests that nonclinician staff may be required to implement express care in STD Clinics. 
Abstract 05-S1.06 Table 1 Agreement between computer-assisted self-interview (CASI) and clinician documentation (Clin) for key clinical and behavioural variables $(\mathrm{N}=875)$

\begin{tabular}{|c|c|c|c|c|c|}
\hline Variable & $\kappa(95 \% \mathrm{CI})$ & CASI+, Clin + (\%) & CASI+, Clin - (\%) & CASI,- Clin $+(\%)$ & CASI-, Clin $-(\%)$ \\
\hline \multicolumn{6}{|l|}{ Reasons for exclusion from express care ${ }^{*}$} \\
\hline Symptoms of STD & $0.67(0.63$ to 0.72$)$ & $436(50)$ & $118(13)$ & $23(3)$ & $298(34)$ \\
\hline Known contact to HIV/STD & $0.60(0.52$ to 0.67$)$ & $91(10)$ & $72(8)$ & $23(3)$ & $689(79)$ \\
\hline Symptomatic sex partner & $0.15(0.05$ to 0.26$)$ & $8(1)$ & $62(7)$ & $10(1)$ & $795(91)$ \\
\hline Positive STD test, needs treatment & $0.12(-0.03$ to 0.27$)$ & $2(0)$ & $28(3)$ & $0(0)$ & $845(97)$ \\
\hline Syphilis in past year & $0.40(0.23$ to 0.72$)$ & $6(1)$ & $13(1)$ & $0(0)$ & $856(98)$ \\
\hline Vaccine indication $†$ & $0.25(0.20$ to 0.31$)$ & $71(8)$ & $249(28)$ & $5(1)$ & $550(63)$ \\
\hline Interested in Plan B $\neq$ & $0.13(-0.04$ to 0.30$)$ & $3(1)$ & $4(2)$ & $25(11)$ & $202(86)$ \\
\hline Interested in contraception $\ddagger$ & $0.32(0.16$ to 0.48$)$ & $15(6)$ & $20(9)$ & $20(9)$ & $166(71)$ \\
\hline \multicolumn{6}{|l|}{ Sensitive behaviours } \\
\hline Male sex partner (lifetime), among men & $0.93(0.90$ to 0.96$)$ & $285(45)$ & $13(2)$ & $10(2)$ & $332(52)$ \\
\hline Injection drug use & $0.82(0.80$ to 0.94$)$ & $43(5)$ & $7(0)$ & $5(0)$ & $820(94)$ \\
\hline Methamphetamine use & $0.71(0.63$ to 0.79$)$ & $68(8)$ & $38(4)$ & $10(1)$ & $759(87)$ \\
\hline $\begin{array}{l}\text { Unprotected anal intercourse with } \\
\text { partners of discordant or unknown } \\
\text { HIV status, among } \mathrm{MSM}^{\S}\end{array}$ & $0.58(0.48$ to 0.68$)$ & $63(22)$ & $35(12)$ & $17(6)$ & $170(59)$ \\
\hline Transactional sex $\neq$ & $0.67(0.50$ to 0.84$)$ & $15(6)$ & $5(2)$ & $8(3)$ & $206(88)$ \\
\hline
\end{tabular}

*Some reasons for exclusion not included here due to lack of comparative data in clinician documentation.

$+\mathrm{Clin}+=$ documented administration or patient declination of HAV, HBV or HPV vaccine.

łLimited to female patients; Clin $+=$ dispensation of Plan B (for Plan B variable) or discussion of contraception plan (for contraception variable).

§Limited to CASI+, Clin+ MSM.

\section{Health services and policy oral session 2-Evaluation of services and policies \\ 05-S2.01 A NATIONAL PROGRAM WITH A NATIONAL IMPACT: QUADRIVALENT HPV VACCINATION AND GENITAL WARTS IN AUSTRALIA, 2004-2010}

doi:10.1136/sextrans-2011-050109.157

\begin{abstract}
1,2B Donovan, ${ }^{1} \mathrm{R}$ Guy, ${ }^{1} \mathrm{H}$ Ali, ${ }^{1} \mathrm{~A}$ Grulich, ${ }^{1} \mathrm{D}$ Regan, ${ }^{1} \mathrm{H}$ Wand, ${ }^{3} \mathrm{C}$ Fairley. ${ }^{1}$ National Centre in HIV Epidemiology and Clinical Research, Sydney; ${ }^{2}$ Sydney Sexual Health Centre, Sydney Hospital, Sydney; ${ }^{3}$ Melbourne Sexual Health Centre and School of Population Health, University of Melbourne, Melbourne, Australia
\end{abstract}

Background From mid-2007 Australia funded a universal free vaccination program for all females between 12 and 26 years, but not for men or for women who were older than 26 years in 2007 . Vaccine coverage rates of $>80 \%$ were achieved for school-girls, though coverage was probably lower for young women in the community. To determine the population effect of the vaccine program we established a national surveillance network to measure trends in clinical presentations for genital warts.

Methods Eight sexual health services dispersed around Australia provided data on all new patients between 2004 and 2010, including new diagnoses of genital warts, demographics, sexual behaviour, and HPV vaccination status.

Results Among more than 130000 new patients we identified over 10000 new cases of genital warts. Before the vaccination program there was no change in the proportion of women or heterosexual men diagnosed with genital warts. In the first 30 months of the vaccination program we detected a $59 \%$ decline in the proportion of young resident women diagnosed with genital warts ( $p$-trend $<0.0001$ ) and preliminary analysis indicates that this trend was ongoing in 2010. In contrast, we could not detect any significant decline in genital warts among non-resident young women, older women, or men who have sex with men. Interestingly, the proportion of younger men ( $<26$ years in mid-2007) diagnosed with genital warts declined by $39 \%$ (p-trend $<0.0001$ ) while there was no significant decline among older heterosexual men. By 2009, $65 \%$ of resident women of free vaccine-eligible age, $15 \%$ of non-resident women of the same age, and $11 \%$ of older women reported having had a quadrivalent or an unknown HPV vaccine.

Conclusion The vaccination program has had a large populationlevel impact on the incidence of genital warts in young Australian women, with some flow-on benefit for young heterosexual men as a result of herd immunity.

\section{5-S2.02 DELAY OF ENTRY INTO CARE IN HIV POSITIVE INDIVIDUALS}

doi:10.1136/sextrans-2011-050109.158

${ }^{1} \mathrm{M}$ van Veen, ${ }^{2} \mathrm{~F}$ de Wolf, ${ }^{3} \mathrm{~T}$ Heijman, ${ }^{2} \mathrm{~S}$ Zaheri, ${ }^{3} \mathrm{H}$ Fennema, ${ }^{4} \mathrm{H}$ Götz, ${ }^{1} \mathrm{M}$ van der Sande. ${ }^{1}$ National Institute for Public Health and the Environment RIVM Bilthoven, Netherlands; ${ }^{2}$ HIV Monitoring Foundation SHM Amsterdam, Netherlands; ${ }^{3}$ Public Health Service, Amsterdam, Netherlands; ${ }^{4}$ Municipal Health Service, Rotterdam-Rijnmond, Netherlands

Background Delay between HIV diagnosis and entry into care may impact not only on the individual prognosis, but hinders interruption of further HIV transmission. Insight into duration and determinants of care-delay and differences between those who do and do not delay are important to further public health policy aimed at reducing ongoing HIV transmission.

Methods (1). Data from the ATHENA national observational cohort for HIV patients with a first date of care from January 2008 until May 2010 were analysed to assess place, date of initial positive diagnosis and entry date into care. (2). Prospective data collection is set up regionally from consenting patients testing HIV positive at the STI clinics in Amsterdam and Rotterdam. Results from February 2009 until April 2010 for time into care and delay are presented. For this analysis delay of entry into care is defined as a time period of 4 weeks or more between confirmed HIV diagnosis and first consultation at the HIV treatment centre.

Results (1). At the national level, $28 \%$ of all new patients entering care $(n=2775)$ was diagnosed HIV positive at their GP, $25 \%$ at an STI clinic, $23 \%$ in the hospital, $2 \%$ at the pregnancy screening, $4 \%$ abroad, $4 \%$ other and 14\% unknown. Median number of days between HIV diagnosis and entry into care was 19 days (IOR 